\title{
THE CLOSURES OF WREATH PRODUCTS IN PRODUCT ACTION
}

\author{
I. PONOMARENKO AND A. V. VASIL'EV
}

\begin{abstract}
Let $m$ be a positive integer and let $\Omega$ be a finite set. The $m$ closure of $G \leq \operatorname{Sym}(\Omega)$ is the largest permutation group on $\Omega$ having the same orbits as $G$ in its induced action on the Cartesian product $\Omega^{m}$. The exact formula for the $m$-closure of the wreath product in product action is given. As a corollary, a sufficient condition is obtained for this $m$-closure to be included in the wreath product of the $m$-closures of the factors.
\end{abstract}

\section{INTRODUCTION}

Let $m$ be a positive integer and let $\Omega$ be a finite set. The $m$-closure $G^{(m)}$ of $G \leq \operatorname{Sym}(\Omega)$ is the largest permutation group on $\Omega$ having the same orbits as $G$ in its induced action on the Cartesian product $\Omega^{m}$. Wielandt 13 , Theorems 5.8 and 5.12] showed that

$$
G^{(1)} \geq G^{(2)} \geq \cdots \geq G^{(m)}=G^{(m+1)}=\cdots=G
$$

for some $m<|\Omega|$. In this sense, the $m$-closure can be considered as a natural approximation of $G$. One can also consider $G^{(m)}$ as the full automorphism group of the family of all $m$-ary relations invariant with respect to $G$.

In general, studying the $m$-closure for $m \geq 2$ is a nontrivial problem both from theoretical and computational point of view, see, e.g., 4, 7, 10, 12. Usual approach here is a reduction via the direct or wreath products to smaller permutation groups. Let us consider these operations in more detail.

Let $K \leq \operatorname{Sym}(\Gamma)$ and $L \leq \operatorname{Sym}(\Delta)$. The direct product $K \times L$ has two natural actions: on the disjoint union $\Gamma \cup \Delta$ and on the Cartesian product $\Gamma \times \Delta$. It is well known that in both cases the $m$-closure of $K \times L$ is equal to $K^{(m)} \times L^{(m)}$ (see, e.g., [2]). A similar formula holds for the wreath product $K \backslash L$ acting on $\Gamma \times \Delta[\underline{6}$. However, passing to the permutation group $K \uparrow L$ induced by the product action of $K$ ? $L$ (i.e., on the Cartesian product of $|\Delta|$ copies of $\Gamma$ ) causes a problem even in a small case:

$$
\begin{aligned}
(\operatorname{Sym}(2) \uparrow \operatorname{Alt}(3))^{(2)} & =\operatorname{Sym}(2) \uparrow \operatorname{Sym}(3), \\
\operatorname{Sym}(2)^{(2)} \uparrow \operatorname{Alt}(3)^{(2)} & =\operatorname{Sym}(2) \uparrow \operatorname{Alt}(3),
\end{aligned}
$$

which shows that, in general, $(K \uparrow L)^{(m)} \not \leq K^{(m)} \uparrow L^{(m)}$. The main goal of the present paper is to establish the exact formula for the $m$-closure of $K \uparrow L$.

The research was supported by the Mathematical Center in Akademgorodok under agreement No. 075-15-2019-1613 with the Ministry of Science and Higher Education of the Russian Federation. 
Apparently, the first results related to the structure of $(K \uparrow L)^{(m)}$ were obtained in [10, Propositions 3.2, 3.3] for the case when the group $K \uparrow L$ is primitive, but even in this case no explicit formula was found. The inclusion $(K \uparrow L)^{(m)} \leq K^{(m)} \uparrow L^{(m)}$ was first proved in [4, Proposition 3.1] for $m=2$ and non 2-transitive $K$ (cf. (2)), and then recently in [8, Theorem 3.3] for $m \geq 3$, primitive $K \uparrow L$, and technical assumptions on the degrees of $K$ and $L$.

To state the main result, we need to define one more closure operator. Namely, for a group $G \leq \operatorname{Sym}(\Omega)$ we denote by $G^{[m]}$ the largest permutation group on $\Omega$ having the same orbits as $G$ in its induced action on the ordered partitions of $\Omega$ in at most $m$ classes. This type of closure behaves similarly to the $m$-closure (cf. formulas (1) and (9) ) and will be considered in more detail in Section 3 .

Theorem 1.1. Let $K$ and $L$ be permutation groups and $m \geq 2$ an integer. Then

$$
(K \uparrow L)^{(m)}=K^{(m) \uparrow L^{[k]},}
$$

where $k=\min \left\{k_{m}, d\right\}$ with $k_{m}=\left|\operatorname{Orb}_{m}(K)\right|$ and $d$ the degree of $L$.

The number $k_{m}$ defined in Theorem 1.1 is bounded from below by the number $\left|\operatorname{Orb}_{m}(\operatorname{Sym}(n))\right|$, where $n$ is the degree of $K$, which is equal to the number of ordered partitions of a set of cardinality $m$ (see, e.g., [8, Example 2.1]). In particular, $k_{m} \geq m+1$ if $m \geq 3$ and $n \geq 2$.

Theorem 1.1 enables us to establish a sufficient condition for the $m$-closure of $K \uparrow L$ to be included in the wreath product of the $m$-closures of $K$ and $L$.

Theorem 1.2. Let $K$ and $L$ be permutation groups and $m \geq 2$. Then

$$
(K \uparrow L)^{(m)} \leq K^{(m)} \uparrow L^{(m)}
$$

unless $m=2$ and $K$ is 2-transitive.

Proof. Without loss of generality, we assume that $K$ is of degree at least 2 . Then $k_{m} \geq m+1$ : this follows from above if $m \geq 3$, and from the fact that $K$ is not 2-transitive if $m=2$. Now if $d \geq m+1$, then $k-1 \geq m$ and Lemma 3.2 together with (11) yields $L^{[k]} \leq L^{(k-1)} \leq \bar{L}^{(m)}$. On the other hand, if $d \leq m$, then $k=d$ and formulas (9) and (11) yield $L^{[\bar{k}]}=L^{[d]}=L \leq L^{(m)}$. Thus in any case, inclusion (4) holds by Theorem 1.1.

Theorem 1.2 gives a natural generalization of the two mentioned results from 4 and 8 . The exceptional case $m=2$ and $K$ is 2 -transitive cannot be avoided, see example in (2). Some other examples of primitive groups $L$ for which $L^{(2)}=L$ and $L^{[2]}>L$ can be found among the groups listed in [11, Theorem 2]. We believe that there are infinitely many such examples where $L$ is imprimitive.

When the group $L$ is primitive and $m \geq 3$, the right-hand side of the equality in Theorem 1.1 can be made more precise with the help of the main results of [11.

Theorem 1.3. Let $K$ and $L$ be permutation groups and $m \geq 3$. Assume that $L$ is primitive and is not an alternating group in standard action. Then

$$
(K \uparrow L)^{(m)}=K^{(m)} \uparrow L .
$$

Proof. Follows from Theorem 1.1 and Lemma 3.3

The authors are grateful to S. V. Skresanov for very useful comments to the first draft of the paper. 


\section{Preliminaries}

We start with some basic facts of Wielandt's theory of $m$-closures. First, note that taking the $m$-closure is a closure operator:

$$
G \leq G^{(m)}, \quad G^{(m)}=\left(G^{(m)}\right)^{(m)}, \quad G \leq H \Rightarrow G^{(m)} \leq H^{(m)},
$$

see [13, Theorem 5.4, 5.9, 5.7], respectively. Second, there is a clear sufficient condition for a permutation to lie in $m$-closure.

Lemma 2.1. (The closure argument, see [13, Theorem 5.6]) Let $G \leq \operatorname{Sym}(\Omega)$, $f \in \operatorname{Sym}(\Omega)$, and $m$ a natural number. Then $f \in G^{(m)}$ if and only if for every $\alpha \in \Omega^{m}$ there is $g \in G$ such that $\alpha^{f}=\alpha^{g}$.

The closure argument is crucial in finding $m$-closures of products of permutation groups, see, e.g., the detailed proof of the following folklore result in [2, Lemma 2.4].

Theorem 2.2. Let $K \leq \operatorname{Sym}(\Gamma), L \leq \operatorname{Sym}(\Delta)$, and let $K \times L$ act on the Cartesian product $\Gamma \times \Delta$. For every integer $m \geq 2$,

$$
(K \times L)^{(m)}=K^{(m)} \times L^{(m)} .
$$

We use the same argument in the proof of our main result for the wreath products of permutation groups in product action. Let us take a closer look at such a product.

Let $K \leq \operatorname{Sym}(\Gamma)$ and $L \leq \operatorname{Sym}(\Delta)$. Without loss of generality, we assume that $\Delta=\{1, \ldots, d\}$. The wreath product $K \backslash L$ induces a permutation group $G=K \uparrow L$ on the Cartesian product

$$
\Omega=\underbrace{\Gamma \times \cdots \times \Gamma}_{d \text { copies }} \text {. }
$$

Every permutation of $G$ can be written in the form

$$
g=\left(g_{1}, \ldots, g_{d} ; \bar{g}\right)
$$

for some $g_{1}, \ldots, g_{d} \in K$ and $\bar{g} \in L$. The action of $g$ on the point

$$
\omega=\left(\omega_{1}, \ldots, \omega_{d}\right) \in \Omega
$$

is defined as follows (see, e.g., [3, Section 2.7]):

$$
\left(\omega^{g}\right)_{i}=\left(\omega_{\bar{i}^{-1}}\right)^{g} \bar{i}^{-1}, \quad 1 \leq i \leq d .
$$

It is well known (see, e.g., [1, Theorem 9.2.1]) that the automorphism group of the Hamming graph is the wreath product of two symmetric groups in product action. When the vertex set of this graph is of the form (5), the edge set is an orbit of $\operatorname{Sym}(\Gamma) \uparrow \operatorname{Sym}(\Delta)$. It almost immediately follows that

$$
(\operatorname{Sym}(\Gamma) \uparrow \operatorname{Sym}(\Delta))^{(2)}=\operatorname{Sym}(\Gamma) \uparrow \operatorname{Sym}(\Delta) .
$$

All undefined notation for permutation groups used in the paper are mostly standard and can be found in 3 . 


\section{Closure With Respect to partitions}

Let $\Omega^{[m]}$ be the set of all ordered partitions $\Pi$ of $\Omega$ such that $|\Pi| \leq m$. For a group $G \leq \operatorname{Sym}(\Omega)$, we denote by $G^{[m]}$ the largest permutation group on $\Omega$ having the same orbits as $G$ in its induced action on $\Omega^{[m]}$. Obviously,

$$
\operatorname{Sym}(\Omega)=G^{[1]} \geq G^{[2]} \geq \cdots \geq G^{[m]}=G^{[m+1]}=\cdots=G
$$

for some $m \leq|\Omega|$.

The groups of series (9), except for the first one, are orbit equivalent to $G$ in the sense of [11], i.e., for all $m \geq 2$,

$$
\operatorname{Orb}\left(G^{[m]}, 2^{\Omega}\right)=\operatorname{Orb}\left(G, 2^{\Omega}\right) .
$$

Indeed, let $S \subseteq \Omega$, and let $\Pi=\left(\Pi_{1}, \Pi_{2}\right)$ the partition of $\Omega$ in two classes $\Pi_{1}=S$ and $\Pi_{2}=\Omega \backslash S$. Now if $H=G^{[m]}$, then $\Pi^{G}=\Pi^{H}$ and hence $S^{G}=\Pi_{1}^{G}=\Pi_{1}^{H}=S^{H}$, as required.

The following statement is an analog of the closure argument (Lemma 2.1).

Lemma 3.1. Let $G \leq \operatorname{Sym}(\Omega), f \in \operatorname{Sym}(\Omega)$, and $m$ a natural number. Then $f \in G^{[m]}$ if and only if for every $\Pi \in \Omega^{[m]}$ there is $g \in G$ such that $\Pi^{f}=\Pi^{g}$.

Proof. The membership $f \in G^{[m]}$ is equivalent to the equality $\left(\Pi^{G}\right)^{f}=\Pi^{G}$ for all $\Pi \in \Omega^{[m]}$, which in turn is equivalent to the existence of $g \in G$ such that $\Pi^{f}=\Pi^{g}$ for each $\Pi \in \Omega^{[m]}$.

Lemmas 2.1 and 3.1 enable us to establish a simple relationship between two types of closures.

Lemma 3.2. $G^{[m+1]} \leq G^{(m)}$.

Proof. Let $g \in G^{[m+1]}$ and $\alpha=\left(\alpha_{1}, \ldots, \alpha_{m}\right) \in \Omega^{m}$. Put $\Pi=\left(\Pi_{1}, \ldots, \Pi_{m+1}\right)$, where $\Pi_{i}=\left\{\alpha_{i}\right\}, i=1, \ldots, m$, and $\Pi_{m+1}=\Omega \backslash\left\{\alpha_{1}, \ldots, \alpha_{m}\right\}$. Then $\Pi \in \Omega^{[m+1]}$. By Lemma 3.1, there exists $h \in G$ such that $\Pi^{g}=\Pi^{h}$. It follows that

$$
\left\{\alpha_{i}^{g}\right\}=\Pi_{i}^{g}=\Pi_{i}^{h}=\left\{\alpha_{i}^{h}\right\}, \quad i=1, \ldots, m .
$$

Thus, $\alpha^{g}=\alpha^{h}$, and we are done by the closure argument.

In general, we cannot improve Lemma 3.2 by replacing $m+1$ by $m$ in $G^{[m+1]}$. Indeed, let $G=\operatorname{Alt}(n), n \geq 3$. Then the stabilizer $G_{1, \ldots, n-2}$ is trivial implying $G^{(n-1)}=G$, see [13, Theorem 5.12]. On the other hand, the group $G$ is $(n-2)$ transitive. Using this, it is not hard to verify that for every ordered partition $\Pi$ with at most $n-1$ classes, $\Pi^{G}=\Pi^{\operatorname{Sym}(n)}$. Together with formula (9), this implies

$$
G^{[m]}= \begin{cases}\operatorname{Sym}(n) & \text { if } m \leq n-1, \\ G & \text { otherwise. }\end{cases}
$$

It follows that $G^{[n-1]} \not \leq G^{(n-1)}$.

Lemma 3.3. Let $G \leq \operatorname{Sym}(n)$ be a primitive group and $m \geq 3$. Then $G^{[m]}=G$, unless $G=\operatorname{Alt}(n)$ and $m \leq n-1$.

Proof. In view of (11), we may assume that $G \nsupseteq \operatorname{Alt}(n)$. The group $G^{[m]} \geq G$ is primitive, and orbit equivalent to $G$, i.e., equality (10) holds. By [11, Corollary 3] this implies that $G=G^{[m]}$, unless

$$
\left(G, G^{[m]}\right) \in \mathfrak{S},
$$


where $\mathfrak{S}$ consists of explicitly described pairs $\left(H, H^{*}\right)$ of primitive groups (of degree at most 10) with $H<H^{*}$.

A straightforward computation in computer package GAP [5] shows that for every pair $\left(H, H^{*}\right) \in \mathfrak{S}$ there is an ordered partition $\Pi=\Pi\left(H, H^{*}\right)$ with three classes such that $H^{*}$ acts on the orbit $\Pi^{H^{*}}$ regularly. Now let $\Pi=\Pi\left(G, G^{[m]}\right)$. Since $|\Pi|=3 \leq m$, we have $\Pi^{G^{[m]}}=\Pi^{G}$. Consequently,

$$
|G| \geq\left|\Pi^{G}\right|=\left|\Pi^{G^{[m]}}\right|=\left|G^{[m]}\right|,
$$

which is possible only if $G^{[m]}=G$.

\section{Proof of Theorem 1.1}

Let $K \leq \operatorname{Sym}(\Gamma)$ and $L \leq \operatorname{Sym}(\Delta)$, where $\Delta=\{1, \ldots, d\}$. Put $G=K \uparrow L$ and $\Omega=\Gamma^{d}$. Then $G \leq \operatorname{Sym}(\Omega)$.

Every $m$-tuple $\alpha \in \Omega^{m}$ is written in the form $\alpha=\left(\alpha^{(1)}, \ldots, \alpha^{(m)}\right)$ where $\alpha^{(j)}=$ $\left(\alpha_{1}^{(j)}, \ldots, \alpha_{d}^{(j)}\right) \in \Gamma^{d}, j=1, \ldots, m$. It is convenient to treat $\alpha$ as a $d \times m$ matrix

$$
\left(\begin{array}{cccc}
\alpha_{1}^{(1)} & \alpha_{1}^{(2)} & \cdots & \alpha_{1}^{(m)} \\
\alpha_{2}^{(1)} & \alpha_{2}^{(2)} & \cdots & \alpha_{2}^{(m)} \\
\cdots & \cdots & \cdots & \cdots \\
\alpha_{d}^{(1)} & \alpha_{d}^{(2)} & \cdots & \alpha_{d}^{(m)}
\end{array}\right)
$$

where the $j$ th column is $\alpha^{(j)}$. In fact, we are rather interested in rows of this matrix which are $m$-tuples of points of $\Gamma$; denote the $i$ th row by $\alpha[i]=\left(\alpha_{i}^{(1)}, \ldots, \alpha_{i}^{(m)}\right)$.

Now let $\operatorname{Orb}_{m}(K)=\left\{s_{1}, \ldots, s_{a}\right\}$, where $a=k_{m}$. For the $m$-tuple $\alpha$, there are uniquely determined numbers $1 \leq a_{1}<\cdots<a_{r} \leq a$ such that $\alpha[i] \in s_{a_{\ell}}$ for every $i \in \Delta$ and some $1 \leq \ell \leq r$. Denote by $\Pi(\alpha)$ the ordered partition of $\Delta$ with classes

$$
\Pi_{\ell}(\alpha)=\left\{i \in \Delta: \alpha[i] \in s_{a_{\ell}}\right\}, \quad \ell=1, \ldots, r .
$$

Note that the integer $k$ from the statement of the theorem is equal to $\min \{a, d\}$.

Lemma 4.1. The mapping $\Omega^{m} \rightarrow \Delta^{[k]}, \alpha \mapsto \Pi(\alpha)$, is well defined and surjective.

Proof. Obviously, $|\Pi(\alpha)| \leq d$ and $|\Pi(\alpha)| \leq a$, and hence $|\Pi(\alpha)| \leq k$ for all $\alpha$. So $\Pi(\alpha) \in \Delta^{[k]}$ and the mapping is well defined. Let $\Pi=\left(\Pi_{1}, \ldots, \Pi_{k}\right) \in \Delta^{[k]}$. Then every $i \in \Delta$ belongs to some $\Pi_{\ell}$; choose arbitrary $\beta_{i} \in s_{\ell}$, which is possible because $k \leq a$. Now let $\alpha$ be the unique $m$-tuple of $\Omega$ for which

$$
\alpha[i]=\beta_{i}, \quad i \in \Delta .
$$

Then $\Pi(\alpha)=\Pi$, as required.

In accordance with formula (77), the permutation $g \in \operatorname{Sym}(\Gamma) \uparrow \operatorname{Sym}(\Delta)$ acts on an $m$-tuple $\alpha$ as follows:

$$
\alpha^{g}[i]=\left(\alpha\left[i^{\bar{g}^{-1}}\right]\right)^{g} i^{\bar{g}^{-1}}, \quad 1 \leq i \leq d .
$$

Thus, $\bar{g}$ permutes the rows of the $d \times m$ matrix $\alpha$, while $g_{i}$ permutes elements of the $i$ th row coordinatewise.

From (11), monotonicity of $m$-closure operator, and (8) it follows that

$$
H=G^{(m)} \leq G^{(2)} \leq(\operatorname{Sym}(\Gamma) \uparrow \operatorname{Sym}(\Delta))^{(2)}=\operatorname{Sym}(\Gamma) \uparrow \operatorname{Sym}(\Delta) .
$$

As in (6), every permutation of $H$ is written in the form

$$
h=\left(h_{1}, \ldots, h_{d} ; \bar{h}\right)
$$


for some $h_{1}, \ldots, h_{d} \in \operatorname{Sym}(\Gamma)$ and $\bar{h} \in \operatorname{Sym}(\Delta)$.

Lemma 4.2. $\Pi(\alpha)^{\bar{h}}=\Pi\left(\alpha^{h}\right)$ for all $h \in K^{(m)} \uparrow \operatorname{Sym}(\Delta)$ and $\alpha \in \Omega^{m}$.

Proof. Let $i \in \Delta$. There is $\ell \in\{1, \ldots, r\}$ such that $i \in \Pi_{\ell}(\alpha)$, i.e., $\alpha[i] \in s_{a_{\ell}}$. By formula (12),

$$
\alpha^{h}\left[i^{\bar{h}}\right]=(\alpha[i])^{h_{i}} \in\left(s_{a_{\ell}}\right)^{h_{i}}=s_{a_{\ell}} .
$$

Let us emphasize that although the indices $a_{\ell}, \ell=1, \ldots, r$, have been defined for the tuple $\alpha$, formula (13) shows that they remain the same for $\alpha^{h}$. Consequently, $i^{\bar{h}} \in \Pi_{\ell}\left(\alpha^{h}\right)$ implying $\Pi_{\ell}(\alpha)^{\bar{h}}=\Pi_{\ell}\left(\alpha^{h}\right)$ for all $\ell$.

Let us prove that $K^{(m)} \uparrow L^{[k]} \leq H$. First, we note that

$$
1 \uparrow L^{[k]} \leq H .
$$

Indeed, let $h \in 1 \uparrow L^{[k]}$ and $\alpha \in \Omega^{m}$. By Lemma 3.1, there exists $\bar{f} \in L$ such that $\Pi(\alpha)^{\bar{h}}=\Pi(\alpha)^{\bar{f}}$. Clearly, $f=(1, \ldots, 1 ; \bar{f}) \in 1 \uparrow L$. By Lemma 4.2

$$
\Pi\left(\alpha^{h f^{-1}}\right)=\Pi(\alpha)^{\bar{h} \bar{f}^{-1}}=\Pi(\alpha) .
$$

It follows that for every $i \in \Delta$, the tuples $\alpha^{h f^{-1}}[i]$ and $\alpha[i]$ belong to the same $m$-orbit of $K$. Therefore,

$$
\alpha^{h f^{-1}}[i]=\alpha[i]^{k_{i}}
$$

for some $k_{i} \in K$. Then $k=\left(k_{1}, \ldots, k_{d}\right) \in K^{d}$ and hence $k f \in K \uparrow L$. Furthermore, formula (15) yields $\alpha^{h f^{-1}}=\alpha^{k}$ implying $\alpha^{h}=\alpha^{k f}$. Thus, $h \in H$ by Lemma 2.1 which proves (14).

By Theorem 2.2. we have $K^{(m)} \uparrow 1=(K \uparrow 1)^{(m)} \leq H$. Together with (14), this yields

$$
K^{(m)} \uparrow L^{[k]}=\left\langle K^{(m)} \uparrow 1,1 \uparrow L^{[k]}\right\rangle \leq H=(K \uparrow L)^{(m)} .
$$

To prove the reverse inclusion, let $h \in H$. For an arbitrary $m$-orbit $s$ of $K$, the set

$$
X_{s}=\left\{\alpha \in \Omega^{m}: \alpha[i] \in s, i=1, \ldots, d\right\}
$$

is invariant with respect to the group $G$. Consequently, $\left(X_{s}\right)^{h}=X_{s}$. By formula (12) this yields

$$
\alpha[i]^{h_{i}}=\alpha^{h}\left[i^{\bar{h}}\right] \in X_{s}
$$

for all $\alpha \in X_{s}$ and $1 \leq i \leq d$. Since $\alpha[i] \in s$, this implies $s^{h_{i}}=s$. Thus, $h_{i}$ preserves each $m$-orbit of $K$ and so $h_{i} \in K^{(m)}$ for all $i$.

It remains to prove that $\bar{h} \in L^{[k]}$ for all $h \in H$. To this end, let $\Pi \in \Delta^{[k]}$. By Lemma 4.1, there is $\alpha \in \Omega^{m}$ such that $\Pi=\Pi(\alpha)$. By the closure argument, there is $g \in K \uparrow L$ such that $\alpha^{h}=\alpha^{g}$. Since $h_{i} \in K^{(m)}$ for all $i$, Lemma 4.2 yields

$$
\Pi^{\bar{h}}=\Pi(\alpha)^{\bar{h}}=\Pi\left(\alpha^{h}\right)=\Pi\left(\alpha^{g}\right)=\Pi(\alpha)^{\bar{g}}=\Pi^{\bar{g}} .
$$

Thus, $\bar{h} \in L^{[k]}$ by Lemma 3.1. It follows that $h \in K^{(m)} \uparrow L^{[k]}$. 


\section{REFERENCES}

[1] A. E. Brouwer, A. M. Cohen, A. Neumaier, Distance-Regular graphs, Springer, Berlin (1989).

[2] D. Churikov, The structure of $k$-closed permutation groups, accepted to Algebra Logic (2021).

[3] J. D. Dixon and B. Mortimer, Permutation Groups, Graduate Texts in Mathematics, 163, Springer (1996).

[4] S. Evdokimov and I. Ponomarenko, Two-closure of odd permutation group in polynomial time, Discrete Math., 235, no. 1-3, 221-232 (2001).

[5] The GAP Group, GAP - Groups, Algorithms, and Programming, Version 4.10.2 (2019), http://www.gap-system.org

[6] L. A. Kalužnin and M. H. Klin, On some numerical invariants of permutation groups, Latv. Mat. Ežegod., 18, no. 1, 81-99 (1976) [in Russian].

[7] M. W. Liebeck, C. E. Praeger, and J. Saxl, On the 2-closures of finite permutation groups, J. London Math. Soc., Ser. 2, 37, no. 2, 241-252 (1988).

[8] E. A. O'Brien, I. Ponomarenko, A. V. Vasil'ev, and E. Vdovin, The 3-closure of a solvable permutation group is solvable, submitted to J. Algebra (2021).

[9] I. Ponomarenko and A. Vasil'ev, Two-closure of supersolvable permutation group in polynomial time, Computational Complexity, 29, 5 (2020).

[10] C. E. Praeger and J. Saxl, Closures of finite primitive permutation groups, Bull. London Math. Soc., 24, 251-258 (1992).

[11] Á. Seress, Primitive groups with no regular orbits on the set of subsets, Bull. London Math. Soc., 29, no. 6, 697-704 (1997).

[12] J. Xu, M. Giudici, C. H. Li, and C. E. Praeger, Invariant relations and Aschbacher classes of finite linear groups, Electron. J. Combin., 18, \#P225 (2011).

[13] H. Wielandt, Permutation groups through invariant relations and invariant functions, The Ohio State University (1969).

Steklov Institute of Mathematics at St. Petersburg, Russia; and Sobolev Institute of Mathematics, Novosibirsk, Russia

Email address: inp@pdmi.ras.ru

Sobolev Institute of Mathematics, Novosibirsk, Russia; and Novosibirsk State University, Novosibirsk, Russia

Email address: vasand@math.nsc.ru 